\title{
Validación al castellano de la Body Responsiveness Connection Scale (Escala de Respuesta Corporal (ERC) en médicos internos del hospital San Rafael de Tunja en Colombia.
}

Spanish validation of the Body Responseveness Connection Scale in medical residents at San Rafael de Tunja Hospital in Colombia.

Juan Carlos Alba Maldonado 1,2,3, Ledmar Jovanny Vargas Rodríguez ${ }^{1,2}$, Gladys Gordillo Navas ${ }^{1,2,3}$.

\section{RESUMEN}

La conexión entre mente y cuerpo hace referencia al proceso de terapias de autorregulación diseñadas para aumentar tanto el bienestar físico como el mental. Objetivo: Determinar las propiedades psicométricas de la Escala de Respuesta Corporal (ERC) en una población de médicos internos y residentes del hospital San Rafael, en la ciudad de Tunja (Colombia). Material y métodos: Se realizó una traducción y retro-traducción de la Body Responseveness Connection Scale. El instrumento fue aplicado a 112 médicos internos del Hospital San Rafael de Tunja, entre 18 y 35 años. Se analizó la consistencia interna del instrumento mediante el test $\alpha$ Cronbach, y la fiabilidad testretest con el coeficiente de correlación intraclase. Resultados: La edad promedio de los participantes fue de 23,5 años ( $\mathrm{DE} \pm 2,06$ años), la mayoría fueron mujeres. El 38.4\% provenían de universidades públicas y el $62,4 \%$ no realizaban actividades adicionales en su tiempo libre. La consistencia interna de la escala fue de 0,87 . El coeficiente de correlación fue mayor de 0,6 en la mayoría de los ítems. Conclusiones: La Escala de Respuesta Corporal en personal de la salud presenta propiedades psicométricas similares a la versión original, con adecuada confiabilidad y validez, factores que permiten evaluar aspectos relevantes de conciencia y disociación corporal.

PALABRAS CLAVE: conciencia, cuerpo, conexión mente cuerpo, escala.

\section{SUMMARY}

The connection between mind and body refers to the process of self-regulation therapies designed to increase both physical and mental well-being. Objective: To determine the psychometric properties of the Body Responsiveness Connection Scale in a population of medical interns and residents at the San Rafael Hospital in the city of Tunja (Colombia). Material and methods: A translation and back translation of the Body Responseveness Connection Scale was performed. The instrument was applied to 112 resident physicians of the San Rafael de Tunja Hospital, between the ages of 18 and 35. The internal consistency of the instrument was analyzed using Cronbach's $\alpha$, and the test-retest reliability with intraclass correlation coefficient. Results: The average age of the participants was 23.5

Departamento de Psiquiatría, Hospital San Rafael. Tunja, Colombia.

2 Facultad de Ciencias de la Salud, Programa de Medicina, Universidad de Boyacá, Tunja, Colombia.

3 Facultad de Ciencias de la Salud, Programa de medicina, Universidad pedagógica y tecnológica de Colombia. Tunja, Colombia. 
years ( $\mathrm{SD} \pm 2.06$ years), the majority were women. $38.4 \%$ came from public universities and $62.4 \%$ reported no additional activities in their free time. The internal consistency of the scale was 0.87 . The correlation coefficient was greater than 0.6 in most of the items. Conclusions: The studied body response scale in health personnel presents psychometric properties similar to the original version, with adequate reliability and validity, which allow the evaluation of relevant aspects of body awareness and dissociation.

KEYWORDS: Body; awareness, responsiveness, body connection scales.

\section{INTRODUCCIÓN}

La conexión mente cuerpo es un término que actualmente se está utilizando en el campo de la medicina, es un término que se utilizaparareferirse al proceso de terapias de autorregulación que están diseñadas para aumentar tanto el bienestar físico como el mental $(1,2)$.

Es importante tener en cuenta que cuando el individuo tiene conocimiento del cuerpo y cómo reacciona ante los diferentes estímulos, así mismo tiene la capacidad de identificar situaciones que afectan de manera perjudicial el equilibrio corporal, este equilibrio es indispensable en el cuidado clínico de los individuos en la recuperación de los traumas psicológicos y/o físicos $(3,4)$.

La "escala de respuesta corporal" (Body Responseveness Connection Scale, BRQ) es una escala desarrollada por Jennifer Daubenmier, investigadora en Psicología Social en la Universidad de California, Berkeley (5); la escala fue diseñada para la investigación y la evaluación de intervenciones empleando terapias corporales (6). La Escala de respuesta Corporal (BRQ) fue creada con el fin de abordar la necesidad de una medida de auto informe para examinar la conciencia corporal y la disociación corporal en las investigaciones mente-cuerpo $(7,8)$.

La capacidad de respuesta corporal de una persona hace referencia a la predisposición que tiene para integrar las sensaciones e imágenes corporales en la conciencia, y de esta forma tomar decisiones y realizar comportamientos en busca de obtener una imagen corporal deseada, la versión en inglés de la escala BRQ encontró que la puntuación total se correlaciona positivamente con la conciencia corporal, el afecto positivo y la práctica cuerpo-mente, según estos hallazgos esta escala es un mediador significativo entre la conciencia corporal y el afecto (5).

El objetivo del estudio fue determinar las propiedades psicométricas de la escala de respuesta corporal en una población de internos del hospital san Rafael en la ciudad de Tunja (Colombia) durante el año 2018.

\section{MATERIAL Y MÉTODOS}

Se realizó un estudio transversal con validación de la escala de respuesta corporal (BRQ), donde se incluyeron en el estudio a todos los médicos Internos del Hospital San Rafael de Tunja, Colombia.

Criterios de inclusión: Ser interno del Hospital San Rafael de Tunja; mayor de 17 años y menor de 35 años. Aceptar participar y firmar electrónicamente el consentimiento informado del estudio.

Criterios de exclusión: Médico general, especialistas y otros profesionales que laboran en la institución; personas mayores de 35 años y menores de 18 años. Personas que no aceptan participar $y / o$ no firman personalmente el consentimiento informado del estudio.

Para la selección de la población se invitó a toda la población de médicos internos de la institución participante, donde se dio una charla previa sobre el motivo de la investigación y los objetivos planteados. Basados en la resolución 8430 de 1993, donde se establecen las normas de investigación en Colombia, se consideró como un estudio sin riesgo, por lo cual se obtuvo el consentimiento informado de cada uno de los participantes.

Para la selección del instrumento se realizó una búsqueda exhaustiva en bases de datos médicocientíficas (Scielo, pubmed, Elsevier, Embase, Scopus), usando como las siguientes palabras clave para la búsqueda: body, awareness, responsiveness, conciencia, cuerpo, conexión mente-cuerpo, body connection scales.

De los resultados encontrados, se seleccionó 
específicamente un artículo en donde se mencionan 32 instrumentos con características psicométricas para la evaluación de la respuesta corporal, entre ellos la escala Body Responseveness Connection Scale ( $\alpha$ Cronbach 0.82) que consta de 7 ítems y evalúa dos dimensiones distintas: la conciencia y disociación corporal.

La escala BRQ original se encuentra en idioma inglés (5), por lo que se realizó una adaptación para el contexto colombiano con una traducción literal de la escala y, posteriormente una traducción en sentido inverso, a continuación se realizó traducción técnica por una profesora de lenguas extranjeras con nivel $\mathrm{C} 1$, posteriormente se efectúo la traducción en sentido inverso por una persona nativa con nivel de inglés $\mathrm{C} 2$ $\mathrm{y}$, finalmente el cuestionario, fue valorado, evaluado y corregido por tres especialistas en psiquiatría (Kappa de Cohen 0.92).

Seguidamente de tener las respectivas traducciones y el cuestionario organizado, se realizó una prueba piloto a 50 estudiantes de medicina de noveno y décimo semestre con el propósito de establecer si este era un instrumento válido, fiable y entendible en una población estudiantil de estudiantes de pregrado. Finalmente, se recibieron algunas sugerencias en cuanto a redacción, dificultad para entender el ítem y palabras con terminología muy científica, por lo tanto se realizaron algunos cambios basados en esto.

El instrumento se aplicóa toda la población en un mismo momento, en el auditorio del Hospital San Rafael. Es una escala fácil de manejar, pues es autoaplicada y tiene una duración máxima de 10 minutos. A cada participante se aplicó la adaptación de la escala que consta de 7 ítems. El ítem 1, 5, 6 y 7 evalúan la conciencia corporal de manera cualitativa desde "no es cierto" (puntaje de 1) hasta es muy cierto (puntaje de 7), teniendo una posibilidad de elegir entre 7 opciones posibles; mientras que las preguntas 2, 3 y 4 evalúan la disociación corporal en forma inversa (Ver anexo 1). Entre más alto es el puntaje para cada subescala, indica que hay una mayor conciencia o una mayor disociación corporal.

Análisis estadístico: Se realizó la validez de apariencia y constructo, en el que se analiza el grado en que el instrumento refleja adecuadamente la teoría subyacente del fenómeno o constructo que se quiere medir. Se estableció por consenso de un grupo de expertos en psiquiatría. El primer paso del análisis fue realizar la prueba de factorabilidad de la escala mediante la prueba de esfericidad de Barrett y el KMO. Posteriormente, se realizó la correlación entre ítems e intraclase. La fiabilidad (repetitividad) del

Anexo 1. Escala traducida y validada al idioma español.

\begin{tabular}{|c|c|c|c|c|c|c|c|c|}
\hline $\mathrm{N}^{\circ}$ & ITEM & $\begin{array}{l}\text { No es } \\
\text { cierto }\end{array}$ & $\begin{array}{l}\text { Muy } \\
\text { poco } \\
\text { cierto }\end{array}$ & $\begin{array}{l}\text { Poco } \\
\text { cierto }\end{array}$ & $\begin{array}{c}\text { A } \\
\text { veces } \\
\text { cierto }\end{array}$ & $\begin{array}{l}\text { Frecuentemente } \\
\text { cierto }\end{array}$ & Cierto & $\begin{array}{l}\text { Muy } \\
\text { cierto }\end{array}$ \\
\hline 1 & $\begin{array}{c}\text { Confió que mi cuerpo me hará saber lo } \\
\text { que es bueno para mí. }\end{array}$ & 1 & 2 & 3 & 4 & 5 & 6 & 7 \\
\hline 2 & $\begin{array}{l}\text { Los impulsos de mi cuerpo me } \\
\text { llevan a hacer cosas de las cuales me } \\
\text { arrepiento. }\end{array}$ & 7 & 6 & 5 & 4 & 3 & 2 & 1 \\
\hline 3 & $\begin{array}{l}\text { Mi mente y mi cuerpo generalmente } \\
\text { quieren hacer dos cosas diferentes. }\end{array}$ & 7 & 6 & 5 & 4 & 3 & 2 & 1 \\
\hline 4 & $\begin{array}{l}\text { Evito las sensaciones y los } \\
\text { sentimientos de mi cuerpo. }\end{array}$ & 7 & 6 & 5 & 4 & 3 & 2 & 1 \\
\hline 5 & $\begin{array}{l}\text { Le hago caso a mi cuerpo cuando me } \\
\text { dice que hacer. }\end{array}$ & 1 & 2 & 3 & 4 & 5 & 6 & 7 \\
\hline 6 & $\begin{array}{l}\text { Para mí es importante saber cómo se } \\
\text { siente mi cuerpo durante el día. }\end{array}$ & 1 & 2 & 3 & 4 & 5 & 6 & 7 \\
\hline 7 & $\begin{array}{c}\text { Disfruto saber cómo se siente mi } \\
\text { cuerpo. }\end{array}$ & 1 & 2 & 3 & 4 & 5 & 6 & 7 \\
\hline
\end{tabular}


instrumento, se analizó mediante el estadístico alfa de Cronbach para conocer la consistencia interna.

\section{RESULTADOS}

En total participaron 112 personas, de los cuales 75 (67\%) eran mujeres y $37(33 \%)$ eran hombres, todos pertenecientes a programas de ciencias de la salud (Medicina, 89,3\% y enfermería, 10,7\%) y cursaban los últimos semestres (octavo $4.5 \%$, noveno $6,3 \%$, onceavo $27,7 \%$, doceavo). Donde el $38,4 \%$ (43 personas) provenían de universidad pública y el $61,6 \%$ (69 personas) de universidad privada.

El promedio de edad fue de 23,5 años ( $\mathrm{DE} \pm 2,06$ años). El $62,4 \%$ refería que no realizaban actividades adicionales en su tiempo libre, el $12,5 \%$ a la lectura de literatura no médica, $12,5 \%$ ver películas, $6,3 \%$ a los video juegos el 1,8\% caminaban, $1,8 \%$ viajaban, $1,8 \%$ jardinería y gimnasio $0,9 \%$.

Mediante el cálculo del análisis factorial se pretende establecer los factores e ítems que subyacen a las condiciones de conciencia y disociación corporal. Se definió la matriz de correlaciones (tabla 1) entre cada uno de los ítems, tendiendo varias con correlación moderada.

Posteriormente, se procedió a realizar un análisis factorial exploratorio (AFE) con los métodos de componentes principales con rotación oblicua mínima -oblimin- con normalización Kaiser. El análisis

Tabla 1. Matriz de correlaciones entre los ítems.

\begin{tabular}{lccccccc}
\hline & ITEM 1 & ITEM 2 & ITEM 3 & ITEM 4 & ITEM 5 & ITEM 6 & ITEM 7 \\
\hline ITEM 1 & 1,000 & & & & & & \\
ITEM 2 & 0,049 & 1,000 & & & & & \\
ITEM 3 & 0,068 & 0,545 & 1,000 & & & & \\
ITEM 4 & $-0,210$ & 0,503 & 0,335 & 1,000 & & & \\
ITEM 5 & 0,426 & 0,135 & 0,123 & $-0,176$ & 1,000 & & \\
ITEM 6 & 0,583 & $-0,068$ & 0,068 & $-0,357$ & 0,503 & 1,000 & \\
ITEM 7 & 0,654 & $-0,249$ & $-0,035$ & $-0,297$ & 0,186 & 0,619 & 1,000 \\
\hline
\end{tabular}

Tabla 2. Descripción de los valores propios y los porcentajes de varianza explicada de los nuevos factores estimados a partir del método de factores comunes.

\begin{tabular}{cccc}
\hline Componente & Total & \% de varianza & \% acumulado \\
\hline 1 & 2,568 & 36,686 & 36,686 \\
2 & 1,818 & 25,970 & 62,656 \\
3 & 0,809 & 11,564 & 74,220 \\
4 & 0,613 & 8,763 & 82,983 \\
5 & 0,541 & 7,728 & 90,711 \\
6 & 0,388 & 5,544 & 96,255 \\
7 & 0,262 & 3,745 & 100,000 \\
\hline
\end{tabular}

Tabla 3. Cargas factoriales encada uno de los ítems

\begin{tabular}{lcc}
\hline & \multicolumn{2}{c}{ Componente } \\
\cline { 2 - 3 } & $\mathbf{1}$ & $\mathbf{2}$ \\
\hline ITEM 1 & 0,775 & \\
ITEM 2 & & 0,846 \\
ITEM 3 & & 0,827 \\
ITEM 4 & & 0,573 \\
ITEM 5 & 0,697 & \\
ITEM 6 & 0,893 & \\
ITEM 7 & 0,658 & \\
\hline
\end{tabular}


de idoneidad de los datos mediante la adecuación muestral de Kaiser-Meyer-Olkin (KMO: 0,826) y una prueba de esfericidad de Barret significativa $(\chi 2$ : 222,04; p 0.000) (tabla 2).

Finalmente se hace la tabla de cargas factoriales encada uno de los ítems luego de la rotación oblicua realizada (tabla 3), con el fin de comprender mejor los resultados se eliminaron de la tabla los resultados que fueran $<0,4$, puesto que son considerados bajos.

Los análisis de correlación se llevaron a cabo con el coeficiente de correlación de Spearman para los dos ítems evaluados, puesto que se trataban de variables categóricas. El coeficiente obtenido para el componente de disociación corporal fue de 0,661 (significativo) y para la conciencia corporal fue de 0,912 (significativo).

La consistencia interna de la escala se evaluó mediante el coeficiente alfa de cronbach considerando aceptable un valor mayor a 0,8 . La escala obtuvo un valor de 0,87 (IC 95\% 0,84-0,90), indicando una adecuada fiabilidad de la prueba.

\section{DISCUSIÓN}

En comparación con otros estudios se evidenció una mayor participación de personas del género masculino $(8,9)$, sin embargo, este es el primer estudio que se realiza en población de personal de la salud.

Los resultados evidencian que la escala de conciencia y disociación corporal, es fiable. El presente instrumento es comprensible para los médicos internos que participaron en el estudio, lo que lo hace útil para valorar la respuesta corporal.

Se ha establecido que esta escala es útil para la valoración y seguimiento del tratamiento psicológico del trauma, trastorno de la conducta alimentaria, obesidad y dolor crónico $(10,11)$, sin embargo, la escalade conciencia podría ayudar en la evaluación de terapias basadas en mindfulness (12). Es importante el presente estudio puestøue por primera vez se ha aplicado esta escala en población hispanohablante y tiene bastante relevancia a nivel médico, pues permite la monitorización de terapias mente cuerpo y en el seguimiento de traumas (abuso físico, sexual y agresión física) $(13,14)$.

El índice de confiabilidad ( $\alpha$ de Cronbach: 0,87 ) nos muestra que este es un instrumento consistente y homogéneo en la valoración que hace en la población estudiada sobre la conciencia y disociación corporal hacialaspersonas con una enfermedad mental. Este dato es similar a lo reportado en la versión original del cuestionario $(\alpha: 0,83)(5)$, a lo encontrado por Quezada $(\alpha: 0,86)(15)$ y por Price y cols $(\boldsymbol{\alpha}: \mathbf{0}, 82)(16), \sin$ embargo, es importante tener en cuenta que estos restudios se realizaron en población general, ninguno se llevó a cabo en personal de la salud, demostrando su validez.

En el presente estudio se determinó que la esfericidad de Barret fue significativa y el test de adecuación de la muestra de KMO fue adecuado. La escala de respuesta corporal (BRQ) es un instrumento estable en el tiempo, puesto que la mayoría de los ítems presentan puntuaciones mayores a 0.6. En un estudio realizado por Gonzalez y cols (17) refiere que en Colombia se realiza poca investigación sobre conciencia corporal, por tanto las escalas que evalúan este ítem no estaban validadas. Adicionalmente, se han establecido algunas escalas para evaluar la imagen corporal en personas con discapacidad física (18), y en población general (19), evaluación de la imagen corporal con la realización de actividad física (20) y el análisis de la imagen corporal comparado con las medidas antropométricas de la población (21).

Una de las limitaciones que se presentan en este estudio es que se realizólavalidaciónen personal de la salud con un límite de edad, por lo cual es importante en futuros estudios evaluar la validez y fiabilidad del instrumento en otras poblaciones. A pesar de ellos, los resultados encontrados son similares a los deotros estudios. En conclusión podemos afirmarque el instrumento es fiable en su versión española. Este estudio es útil por diferentes motivos. El primero de ellos es que permite que dispongamos de la validación en español de un instrumento necesario para comprender la respuesta corporalante distintas situaciones, y en segundo lugar, permitirá que se realicen estudios descriptivos poblaciones sobre la conciencia y la disociación corporal. Y en tercer lugar, esta comprensión de la situación facilitará que se realicen intervenciones adecuadas para tratar las personas con patologías mentales.

\section{CONCLUSIONES}

La escala de respuesta corporal en personal de la salud presenta propiedades psicométricas similares a la versión original, con adecuada confiabilidad y validez, con lo cual nos permite evaluar la conciencia y la disociación corporal. 
Agradecimientos: A los médicos internos del Hospital San Rafael de Tunja y a los estudiantes de psiquiatria de la Universidad de Boyacá por su participación en la investigación.

\section{Correspondencia:}

Ledmar Jovanny Vargas Rodríguez

Correo electrónico: lejovaro@gmail.com

Conflictos de interés: Ninguno.

Financiación: Ninguna.

\section{REFERENCIAS BIBLIOGRÁFICAS.}

1. Brytek-Matera A, Kozieł, A. The body self-awareness among women practicing fitness: a preliminary study. Polish Psychological Bulletin. 2015; 46(1): 104-111

2. Ceunen E, Van Diest I, Vlaeyen J . Accuracy and awareness of perception: related, yet distinct (commentary on Herbert et al., 2012). Biological Psychology. 2013; 92(2): 426-427. Doi: 10.1016/j. biopsycho.2012.09.012

3. Daubenmier JJ, Sze J, Kerr CE, Kemeny ME, Mehling W. Follow your breath: respiratory interoceptive accuracy in experienced meditators. Psychophysiology. 2013; 50(8): 777-789. Doi: 10.1111/psyp. 12057

4. Dittmann KA, Freedman MR. Body awareness, eating attitudes, and spiritual beliefs of women practicing yoga. Eating Disorders. 2009; 17(4): $273-$ 292. Doi: $10.1080 / 10640260902991111$

5. Tihanyi E, Ferentzi E, Daubenmier J, Drew R, Köteles F. Body responsiveness questionnaire: Validation on a european sample, mediation between body awareness and affect, connection with mindfulness, Body Image, and Physical Activity. International Body Psychotherapy Journal. 2016; 16 (1): 56-73.

6. Daubenmier JJ, Kristeller J, Hecht FM, et al. Mindfulness intervention for stress eating to reduce cortisol and abdominal fat among overweight and obese women: An exploratory randomized controlled study. Journal of Obesity. 2011; 15 (2): 105 - 112. Doi: $10.1155 / 2011 / 651936$

7. Carmody J, Baer RA. Relationships between mindfulness practice and levels of mindfulness, medical and psychological symptoms and well-being in a mindfulness-based stress reduction program. J Behav Med. 2008;31:23-33.

8. Afrell M, Biguet G, Rudebeck CE. Living with a body in pain - between acceptance and denial. Scand J Caring Sci. 2007;21:291-6.

9. Christensen AJ, Wiebe JS, Edwards DL, Michels JD, Lawton WJ. Body consciousness, illness-related impairment, and patient adherence in hemodialysis. J Consult Clin Psychol. 1996; 64:147- 52.
10. Eriksson EM, Möller IE, Söderberg RH, Eriksson HT, Kurlberg GK. Body awareness therapy: A new strategy for relief of symptoms in irritable bowel syndrome patients. World J Gastroenterology. 2007; 13:3206-14.

11. Brown K, Ryan R. The benefits of being present: Mindfulness and its role in psychological wellbeing. J Pers Social Psychol. 2003;84:822-48.

12. Gard G. Body awareness therapy for patients with fibromyalgia and chronic pain. Disabil Rehabil. 2005;27:725-8.

13. Mehling WE, Hamel KA, Acree M, Byl N, Hecht FM. Randomized, controlled trial of breath therapy for patients with chronic lowback pain. Altern Ther Health Med. 2005;11:44-52.

14. Cramer H, Lauche R, Daubenmier J, Mehling W, Bussing A, Saha FJ, et al. Being aware of the painful body: Validation of the German Body Awareness Questionnaire and Body Responsiveness Questionnaire in patients with chronic pain. PLoS ONE. 2018; 13(2): e0193000. DOI: 10.1371/journal. pone. 019300

15. Quezada-Berumen L, González-Ramírez M, Cebolla A, Soler J, Garcia-Campayo J. Conciencia corporal y mindfulness: Validación de la versión española de la escala de conexión corporal (SBC). Actas Esp Psiquiatr. 2014;42(2):57-67.

16. Price C, Thompson E. Measuring Dimensions of Body Connection: Body Awareness and Bodily Dissociation. J Altern Comp Med. 2007;13:945-53.

17. Sanabria O, Camacho P. Yoga y danza integrada como estrategias dirigidas a la conciencia corporal en amputados de la fuerza publica. Rev psicoterap. 2017; 28 (108): 209-227.

18. Botero PA, Londoño Pérez, C. Diseño y validación de un cuestionario de imagen corporal para personas en situación de discapacidad física. Revista Colombiana de Psicología. 2015; 24(1): 219-233. doi:10.15446/rcp.v24n1.45644

19. Schaefer L, Thompson K. The development and validation of the Physical Appearance Comparison Scale-3 (PACS-3). Psychol Assess. 2018; 30(10):1330-1341. doi: 10.1037/pas0000576.

20. Menzel J, Thompson J, Levine M. Development and validation of the Physical Activity Body Experiences Questionnaire. Bull Menninger Clin. 2019;83(1):5383. doi: 10.1521/bumc.2019.83.1.53

21. Pellizzer ML, Tiggemann M, Waller G, Wade TD. Measures of body image: Confirmatory factor analysis and association with disordered eating. Psychological Assessment. 2018; 30(2): 143-153. DOI: 10.1037/ pas0000461

Recibido: 29/07/2020

Aceptado: 12/07/2021 